Case Report

\title{
Successful Surgical Repair of Scimitar Syndrome in a 38-Year-Old Adult
}

\author{
Rafael Meza ${ }^{1}$, John Araujo ${ }^{2,}$, , Alejandro Escobar ${ }^{1}$, Alejandra Echeverri ${ }^{3}$, Juan Turizo ${ }^{1}$, \\ Susana Cardona ${ }^{1}$ \\ ${ }^{1}$ Department of Congenital Heart Surgery, Somer in Care Cardiovascular Center, Rionegro, Colombia \\ ${ }^{2}$ Department of Pediatric and Adult Congenital Heart Disease, Somer in Care Cardiovascular Center, Rionegro, Colombia \\ ${ }^{3}$ Department of Pediatric and Adult Cardiovascular Anesthesiology, Somer in Care Cardiovascular Center, Rionegro, Colombia
}

Email address:

johnjairoaraujo@gmail.com (J. Araujo)

${ }^{*}$ Corresponding author

\section{To cite this article:}

Rafael Meza, John Araujo, Alejandro Escobar, Alejandra Echeverri, Juan Turizo, Susana Cardona. Successful Surgical Repair of Scimitar Syndrome in a 38-Year-Old Adult. International Journal of Cardiovascular and Thoracic Surgery. Vol. 5, No. 6, 2019, pp. 80-83. doi: $10.11648 /$ j.ijcts.20190506.12

Received: November 30, 2019; Accepted: December 19, 2019; Published: January 7, 2020

\begin{abstract}
Scimitar syndrome is an infrequent congenital malformation. It is a partial anomalous pulmonary venous drainage of the right lung into the inferior vena cava. Up to $25 \%$ of cases are associated with other heart diseases. The incidence in adults is reported to be $0.5-0.7 \%$; classically, the adult form does not have pulmonary arterial hypertension, and may be unnoticed and asymptomatic for many years. It often presents after the second decade of life with nonspecific symptoms. Without surgical repair, clinical worsening, increased pulmonary pressure and sustained pulmonary arterial hypertension ultimately occur, when the syndrome is associated with other congenital heart diseases. We present the case of a 38-year-old woman diagnosed with scimitar syndrome associated with a large superior sinus venosus atrial septal defect, who underwent successful surgical repair using a lateral pericardial tunnel technique to redirect right pulmonary venous flow to the left atrium through a left atriotomy. This repair has shown good medium-term results. Possible complications such as tunnel thrombosis and stenosis will need to be explored in future assessments, and follow up must be lifelong.
\end{abstract}

Keywords: Scimitar Syndrome, Partial Anomalous Pulmonary Venous Drainage, Adult Congenital Heart Disease, Pericardial Tunnel

\section{Introduction}

Scimitar syndrome, a rare and complex congenital malformation with an incidence of 2 per 100,000 live births [1], was first described in 1836 [2]. This condition was typified by partial anomalous pulmonary venous drainage of the right lung into the inferior vena cava (IVC) and the term was coined by Neill et al. in 1960 [3]. The pulmonary vein may descend caudally towards the diaphragm in a crescent shape and curve sharply just above or below the IVC right atrial junction, producing the classic scimitar sign similar to a Turkish sword [4]. It affects women more than men. Three clinical forms have been described: infantile, adult and associated with multiple malformations.
The infantile form is the most severe, manifesting in the first months of life with signs of congestive heart failure, recurrent pneumonia, and progressive pulmonary arterial hypertension (PAH) [5]. The adult form has a better prognosis, and is not associated with $\mathrm{PAH}$. The affected lung develops various degrees of hypoplasia and pulmonary artery malformations. Up to $25 \%$ of cases are associated with other heart diseases such as coarctation of the aorta, tetralogy of Fallot, patent ductus arteriosus, dextrocardia and atrial septal defect (ASD). Pulmonary malformations such as horseshoe lung, accessory diaphragm and bronchogenic cysts, among others, are also associated with this syndrome $[6,7]$.

We present the case of a 38-year-old woman diagnosed with 
scimitar syndrome who underwent successful surgical repair using a lateral pericardial tunnel technique which redirected the right pulmonary venous flow to the left atrium through a left atriotomy.

\section{Case Report}

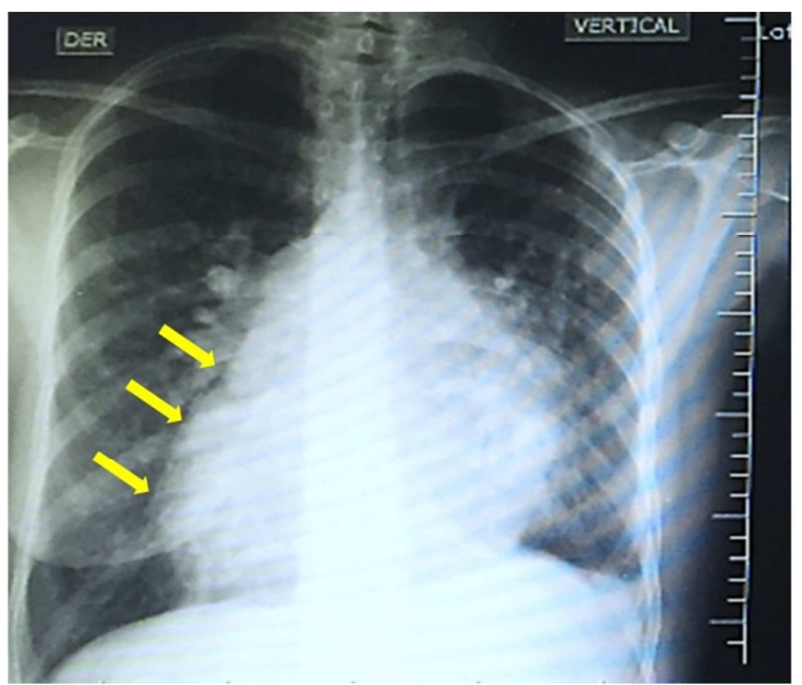

Figure 1. Chest $x$-ray shows cardiomegaly and a right paracardiac descending opacity (yellow arrows), corresponding to a collector driving downwards and draining towards the inferior vena cava.

A 38-year-old woman consulted her physician due to worsening dyspnea and functional class over the last year. She was referred to the adult congenital heart disease service at our center, with no significant medical history or previous hospitalizations. On physical exam, she had a regular heart rhythm, increased right sternal precordial activity, a fixed split second heart sound, a right midsternal systolic murmur, symmetrical pulses, no peripheral edema and $94 \%$ saturation on room air. The electrocardiogram showed a normal sinus rhythm, $+120^{\circ} \mathrm{QRS}$ right axis deviation, right chamber enlargement and right bundle branch block. The chest $\mathrm{x}$-ray revealed cardiomegaly, with right contour enlargement, an upward tilted apex as an indirect sign of right ventricular (RV) enlargement, and right paracardiac descending opacity (Figure 1). The transesophageal echocardiogram showed a dilated RV (basal diameter $50 \mathrm{~mm}$ ), moderate hypokinesia of the free wall (TAPSE $15 \mathrm{~mm}$ ), a severely dilated right atrium $\left(46 \mathrm{~cm}^{2}\right.$ area), a $20 \mathrm{~mm}$ superior sinus venosus ASD with a left to right shunt, a dilated pulmonary artery (38 mm trunk) and a 60\% left ventricular ejection fraction. The return and connection of the left veins was adequately identified, but the arrival of the right veins could not be seen. In light of the right paracardiac image and the superior sinus venosus ASD, a partial anomalous venous return of the right pulmonary veins to the inferior vena cava (scimitar syndrome) was suspected. A complementary cardiac resonance was performed which showed partial abnormal pulmonary venous return of the right pulmonary veins through a collector driving downwards and draining towards the IVC (intrathoracic portion), without stenosis or pulmonary sequestration, associated with a $20 \mathrm{~mm}$ superior sinus venosus ASD. QP: 4.2 and QS: 1 were calculated (Figure 2).

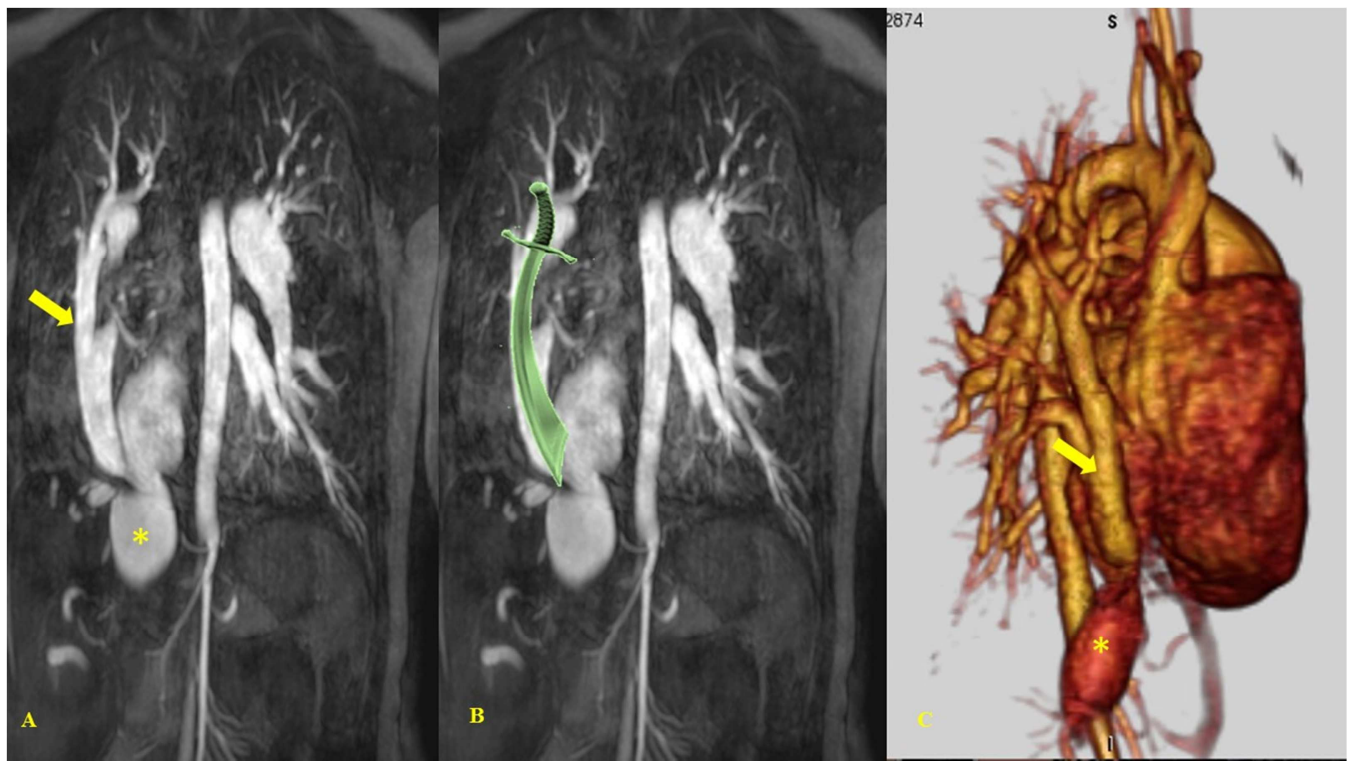

Figure 2. A. Cardiac resonance shows partial anomalous pulmonary venous return of the right pulmonary veins through a collector (yellow arrow) driving downwards and draining towards the inferior vena cava (*). B: Comparative image, showing the classical Turkish sword sign. C: A $3 D$ reconstruction; shows the same as in $A$.

Finally, a cardiac catheterization was carried out showing a mean pulmonary pressure of $40 \mathrm{mmHg}$, a pulmonary vascular resistance index (PVRI) of 4 Wood units $/ \mathrm{m}^{2}$, and a systemic vascular resistance index (SVRI) of 22 Wood units $/ \mathrm{m}^{2}$, (PVRI/SVRI index: 0.18), under basal conditions.
According to the new 2018 anatomic and physiological (AP) classification of adults with congenital heart disease (ACHD) [8], she was classified as AP IIC. Surgical repair was undertaken: arterial cannulation was performed at the ascending aorta and venous cannulation was executed with a 
single cannula at the right atrium. After establishing cardiopulmonary bypass and cooling the patient, she was kept under hypothermic circulatory arrest at $20.8^{\circ} \mathrm{C}$ for 44 minutes (clamp and perfusion times: 80 and $102 \mathrm{~min}$, respectively). Inspection inside the IVC confirmed the orifice of this anomalous pulmonary vein to be in the posterior wall of the IVC (Figure 3). The atrial septectomy was performed with complete excision of the floor of the fossa ovalis. Next, the length of the intra-atrial baffle was trimmed and sutures were placed around the perimeter of the atrial septectomy, ensuring that pulmonary venous blood from the right lung via the anomalous veins was now directed underneath the baffle, back to the left atrium (Figure 4).

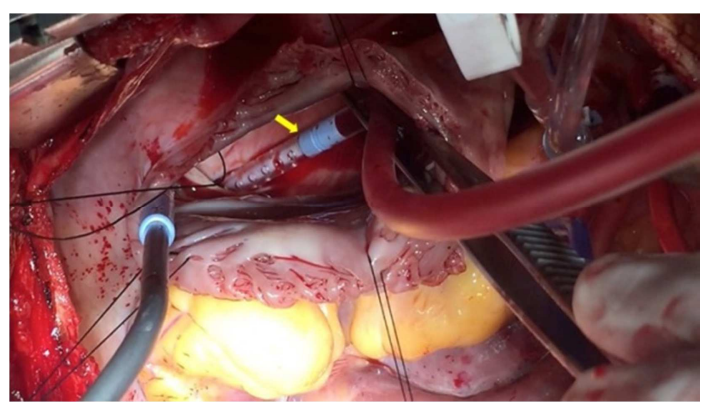

Figure 3. The right atrium is open and the tip of the sucker (yellow arrow) is located on the posterior wall of the inferior vena cava where the orifice of the right anomalous pulmonary drainage is found.

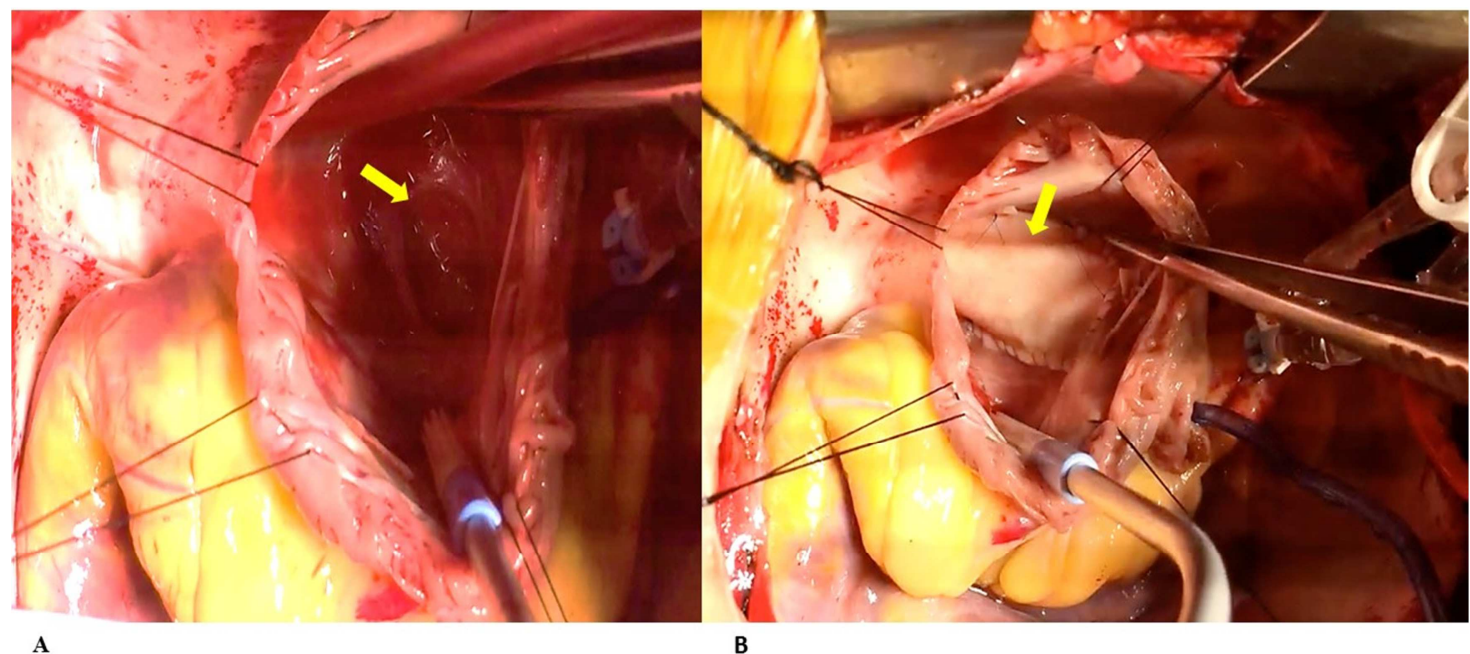

Figure 4. A. The right atrium is open, with the yellow arrow indicating the superior sinus venosus atrial septal defect. B. Intra-atrial baffle with pericardial patch (yellow arrow) which redirected the right pulmonary venous flow to the left atrium.

\section{Result}

The patient was discharged seven days after surgery, without complications. She was managed medically with warfarin. Follow up at one year showed that the size of the right heart chambers had normalized and pulmonary pressure was normal. She was reclassified as AP class IA.

\section{Discussion}

Scimitar syndrome, in its classic or complete form, is made up of a partial anomalous connection of the right pulmonary veins to the suprahepatic portion of the IVC, dextroposition of the heart due to dextrorotation, hypoplasia of the right lung, hypoplasia of the right pulmonary artery, systemic anomalies of the right lung arterial irrigation (aortopulmonary collaterals), bronchopulmonary sequestration with agenesis of the right upper or middle bronchus, and, in up to $25 \%$ of cases, other extracardiac and cardiac anomalies.

The adult form does not have PAH, and may be unnoticed and asymptomatic for many years, as in this case. It occurs infrequently in adults, with a reported incidence of $0.5-0.7 \%$ $[9,10]$. It often presents after the second decade of life with nonspecific symptoms such as diminished exercise tolerance, mild dyspnea with exercise, supraventricular arrhythmias, recurrent respiratory infections, and complicated pneumonia, among others [11]. The classic radiological findings include cardiac dextroposition, and the scimitar sign may be present in many studies (Figure 1). In cases without associated anomalies, with scant left to right flow (Qp: < 1.4; Qs: 1), surgical repair is not necessary [12]. In the current case, the associated large superior sinus venosus ASD led to increased hemodynamic repercussions and was one reason for repair. The presence of a sinus venosus ASD, by itself, increases the risk of $\mathrm{PAH}$ by up to $16 \%$, compared to individuals with ostium secundum defects (4\%) [13].

Without surgical repair, clinical worsening, increased pulmonary pressure and the development of sustained PAH were inevitable. Following the recommendations and guidelines for surgical repair in ACHD [14], this case fulfilled the surgical safety criteria for repair. The intrapericardial tunnel surgical technique has shown good medium-term results in the cases reported to date [15]. Possible complications such as tunnel thrombosis and stenosis will need to be explored in future assessments and follow up [16]. The pericardial tunnel technique has shown good medium-term results. Follow up must be lifelong and should be aimed at detecting the potential complications described. 


\section{Conclusions}

Scimitar syndrome is a rare congenital malformation in adults, and although it has a better prognosis than the infantile forms, it continues to be a diagnostic challenge for cardiologists. The association with other congenital defects, such as in the presented case, increases the degree of hemodynamic repercussion, making surgical repair mandatory. The decision to undertake surgical repair must be well founded, following the ACHD management recommendations. The pericardial tunnel technique has shown good medium-term results. Follow up must be lifelong and should be aimed at detecting the potential complications described.

\section{Consent}

The patient gave informed written consent for this publication.

\section{Conflicts of Interest}

None of the authors have any possible conflicts of interest.

\section{References}

[1] Wu Y, Wu Z, Zheng J, Li Y, Zhou Y, Kuang H, et al. Sutureless technique versus conventional surgery in the primary treatment of total anomalous pulmonary venous connection: a systematic review and meta-analysis. J Cardiothorac Surg. 2018; 13 (1): 69.

[2] Luna A, González G, Echeverry P. Scimitar syndrome and anesthetic implications. Rev Colomb Anestesiol. 2015; 43 (3): 245-49.

[3] Youssef T, Mahmoud H, Ionescu N, Stoica D, Grigore C, Nicolescu A, et al. Scimitar syndrome associated with aberrant right subclavian artery, diaphragmatic hernia, and urinary anomalies - case report and review of the literature. Romanian J Morphol Embryol Rev Roum Morphol Embryol. 2018; 59 (2): 625-30.

[4] Masrani A, McWilliams S, Bhalla S, Woodard P. Anatomical associations and radiological characteristics of Scimitar syndrome on CT and MR. J Cardiovasc Comput Tomogr. July 2018; 12 (4): 286-9.

[5] Al Rukban H, Al Ghaihab M, Tamimi O, Al-Saleh S. Clinical spectrum of infantile scimitar syndrome: A tertiary center experience. Ann Pediatr Cardiol. 2014; 7 (1): 29-33.

[6] Espinola N, Játiva S, Muñoz L, Zamora C. Aspectos clínicos y ecocardiográficos del síndrome de la cimitarra. Rev Esp Cardiol. 2006; 59 (3): 284-8.

[7] Vida V, Padrini M, Boccuzzo G, Agnoletti G, Bondanza S, Butera G, et al. Natural History and Outcome of "Uncorrected" Scimitar Syndrome Patients: A Multicenter Studyof the Italian Society of Pediatric Cardiology. Rev Esp Cardiol. 2013; 66 (7): 556-60.

[8] Stout K, Daniels C, Aboulhosn J, Bozkurt B, Broberg C, Colman J, et al. 2018 AHA/ACC Guideline for the management of adults with congenital heart disease: executive summary: a report of the American College of Cardiology/American Heart Association Task force on clinical practice guidelines. J Am Coll Cardiol. 2019; 73 (12): 1494-63.

[9] Vida V, Padalino M, Boccuzzo G, Tarja E, Berggren H, Carrel T, e al. Scimitar syndrome A European Congenital Heart Surgeons Association (ECHSA) Multicentric Study. Circulation. 2010; 122: 1159-166.

[10] Dusenbery S, Geva T, Seale A, Valente A, Zhou J, Sena L, et al. Outcome predictors and implications for management of scimitar síndrome. Am Heart J. 2013; 165 (5): 770-7.

[11] Kahrom M, Kahrom H. Scimitar syndrome and evolution of managements. The Pan African Medical Journal. 2009; 3: 20.

[12] Warnes C, Williams R, Bashore T, Bashore T, Child J, Conolly $\mathrm{H}$, et al. ACC/AHA 2008 Guidelines for the managent of adults with congenital heart disease. J Am Coll Cardiol. 2008; 52 (23): 143-63.

[13] Galie N, Manes A, Palazzini M, Negro L, Marinelli A, Gambetti S, et al. Management of pulmonary arterial hypertension associated with congenital systemic-to-pulmonary shunts and Eisenmenger's syndrome. Drugs 68: 1049-1066.

[14] Galiè N, Humbert M, Vachiery J, Gibbs S, Lang I, Torbicki A, et al. 2015 ESC/ERS Guidelines for the diagnosis and treatment of pulmonary hypertension: The Joint Task Force for the Diagnosis and Treatment of Pulmonary Hypertension of the European Society of Cardiology (ESC) and the European Respiratory Society (ERS): Endorsed by: Association for European Paediatric and Congenital Cardiology (AEPC), International Society for Heart and Lung Transplantation (ISHLT). Eur Heart J. 2016; 37 (1): 67-119.

[15] Sun Y, Zhang H, Liu J, Xu Z, Wang S, Zhu H. Pericardial tunnel technique in the surgical management of the vertical form of scimitar syndrome. Interact Cardiovasc Thorac Surg. 2018; 27 (3): 387-93.

[16] Benito F, González A, Oliver J. Intraluminal dilation of inferior vena cava stenosis after repair of the scimitar syndrome in an adult patient. Rev Esp Cardiol 2002; 55 (2): 190-2. 